\title{
Grayscale-to-Color: Scalable Fabrication of Custom Multispectral Filter Arrays
}

\author{
Calum Williams, ${ }^{*}, \dagger, \ddagger$ George S. D. Gordon, ${ }^{\dagger}$ Timothy D. Wilkinson, ${ }^{\dagger}$ and Sarah E. Bohndiek ${ }^{*}, \S_{\odot}$ \\ ${ }^{\dagger}$ Electrical Engineering Division, Department of Engineering, University of Cambridge, $9 \mathrm{JJ}$ Thomson Avenue, Cambridge, CB3 \\ OFA, U.K. \\ ${ }^{\ddagger}$ Department of Physics, Cavendish Laboratory, University of Cambridge, JJ Thomson Avenue, Cambridge, CB3 OHE, U.K. \\ ${ }^{\S}$ Cancer Research UK Cambridge Institute, University of Cambridge, Robinson Way, Cambridge, CB2 ORE, U.K.
}

\section{Supporting Information}

ABSTRACT: Snapshot multispectral image (MSI) sensors have been proposed as a key enabler for a plethora of multispectral imaging applications, from diagnostic medical imaging to remote sensing. With each application requiring a different set, and number, of spectral bands, the absence of a scalable, cost-effective manufacturing solution for custom multispectral filter arrays (MSFAs) has prevented widespread MSI adoption. Despite recent nanophotonic-based efforts, such as plasmonic or high-index metasurface arrays, large-area MSFA manufacturing still consists of many-layer dielectric (Fabry-Perot) stacks, requiring separate complex lithography steps for each spectral band and multiple material compositions for each. It is an expensive, cumbersome, and inflexible undertaking, but yields optimal optical performance. Here, we demonstrate a manufacturing process that enables cost-effective wafer-level fabrication of custom MSFAs in a single lithographic step, maintaining high efficiencies $(\sim 75 \%)$ and narrow line widths $(\sim 25 \mathrm{~nm})$ across the visible to near-infrared. By merging grayscale (analog) lithography with metal-insulator-metal (MIM) Fabry-Perot cavities, whereby exposure dose controls cavity thickness, we demonstrate simplified fabrication of MSFAs up to $N$-wavelength bands. The concept is first proven using low-volume electron beam lithography, followed by the demonstration of large-volume UV mask-based photolithography with MSFAs produced at the wafer level. Our framework provides an attractive alternative to conventional MSFA manufacture and metasurface-based spectral filters by reducing both fabrication complexity and cost of these intricate optical devices, while increasing customizability.

KEYWORDS: nanophotonics, multispectral, optical sensor, microfabrication, color filter arrays
$\mathrm{C}$ omplementary metal-oxide-semiconductor (CMOS) image sensors are low cost and compact, implemented in a plethora of applications from digital photography to medical imaging. ${ }^{1-3}$ To resolve wavelength-specific information, spatially variant and spectrally distinct color filter arrays (CFAs) are deposited in mosaic-like patterns atop the image sensor with a pitch matched to the pixel size. The most widespread CFA is the Bayer filter, ${ }^{4}$ which consists of red, green, and blue (RGB) color filters to replicate human vision. In recent years, more complex mosaics incorporating additional wavelength filters (spectral bands) referred to as multispectral filter arrays (MSFAs) have been proposed to enable multi- and hyperspectral imaging systems. ${ }^{2,3,5,6}$ Typically, each multispectral imaging (MSI) application requires a specific spectral range and number of spectral bands; however, a cost-effective "one size fits all" manufacturing approach remains elusive. . $^{2,3,5,7,8}$ The absence of a scalable, cost-effective manufacturing solution is preventing the widespread adoption of MSI to meet the current demand in exciting applications ranging from remote sensing to biomedical imaging.

Conventional CFAs/MSFAs are manufactured with either absorptive dyes, one for each wavelength, or multilayer FabryPerot (FP) cavities, comprising a different combination of alternating dielectric materials for each wavelength. ${ }^{3,9-11}$ Both approaches are cumbersome from a fabrication point of view: the FP approach (structural color) requires $N$-lithographic/ hard-mask steps and $N$ different stack architectures to achieve $N$ spectral bands, yet provides excellent narrowband optical performance and is widely implemented commercially. The FP cavity provides superior narrowband optical performance, yet for MSI applications that require custom MSFAs, the costeffective manufacture of high-performance, arbitrary spectral band mosaics is challenging. Alternate methodologies for

Received: August 20, 2019

Published: October 23, 2019 


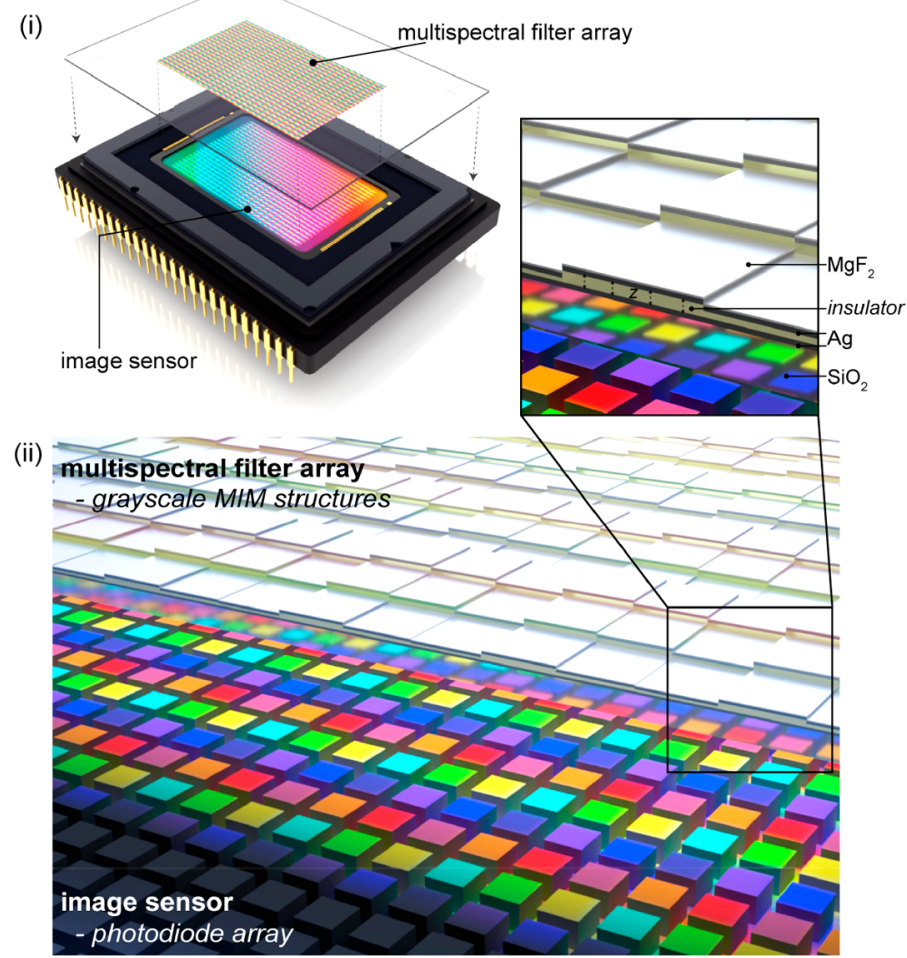

b

(i)

(ii)

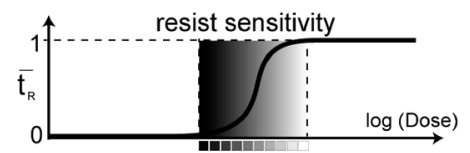

(iii)

(iv)

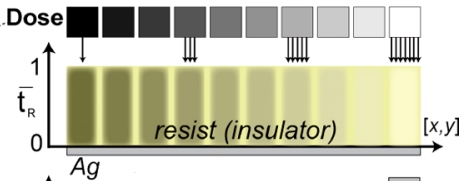

(v)

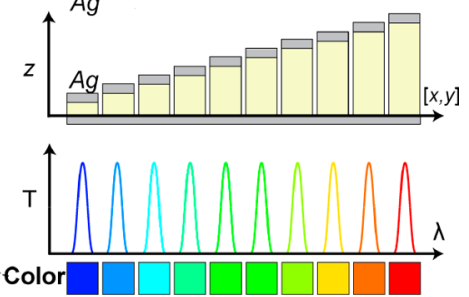

Figure 1. Multispectral filter arrays (MSFAs) using grayscale lithography with metal-insulator-metal (MIM) geometry. (a) Schematic: (i) using a customized MSFA atop a monochrome image sensor for multispectral imaging. (ii) 3D MIM structure of MSFA with inset detailing layers. The wavelength transmitted to each pixel below the MIM structures is controlled with the single-step lithographic fabrication process. (b) MSFA fabrication process: (i) a spatially varying grayscale exposure dose results in a spatially varying wavelength transmission profile. (ii) Calculated grayscale exposure dose profile corresponding to remaining resist thickness profiles ("resist sensitivity" curve). An ultrathin noble metal (Ag) layer on glass $\left(\mathrm{SiO}_{2}\right)$ acts both to dissipate accumulated charge and as the bottom mirror of the filter. (iii) A spatially variant dose modulated exposure leaves a 3D resist profile postdevelopment. (iv) Post-metal-deposition: with a top metal (mirror) layer, the spatially varying 3D resist profile acts to filter the light according to the eigenmode solution of the stack. (v) Final spectral transmittance profiles of MIM structures.

spectral filtering have been proposed, including plasmonic arrays, ${ }^{10,12-21}$ high-index dielectric metasurfaces, ${ }^{2,23}$ diffractive elements, ${ }^{24}$ and ultrathin dielectric coatings. ${ }^{25}$ Such approaches overcome the need for multiple lithographic steps, but typically present additional problems: inherent polarization sensitivity due to $1 \mathrm{D} / 2 \mathrm{D}$ grating unit cells; low transmission efficiencies, either through plasmonic losses or operating with linear polarization states; often infrared rather than visible spectral responses; broad full-width at halfmaximums (fwhm's), yielding poor wavelength selectivity; many higher-order lattice-based resonances; and require expensive ultrahigh resolution (non-industry standard) lithographic patterning for commercialization.

Metal-insulator-metal (MIM) cavities-a metallic form of the FP cavity ${ }^{26,27}$ - have been widely shown to provide comparable high transmission efficiency narrowband performance, without the requirement for many dielectric layers. ${ }^{26-30}$ MIM-based filters have been proposed for simpler CFA stack architectures; ${ }^{31-34}$ however, as with the all-dielectric approach, each cavity height (spectral band) requires a separate lithographic step. A potential solution to this is to utilize analog lithographic techniques to control FP cavity height. Early work suggests this is a promising approach, with reflective $^{35,36}$ and transmissive ${ }^{37}$ MIM pixel arrays recently demonstrated. Unfortunately, for practical realization these preliminary approaches are inadequate; they use nonscalable, direct-write electron beam lithography (EBL) over remarkably small lateral areas and provide relatively poor optical performance that falls short of state-of-the-art MSFAs. Moreover, "subpixel" elements are utilized, with resultant lattice periods and effective fill factors. ${ }^{35-37}$ As a result, their transmission efficiency is limited, additional diffractive orders are introduced along with polarization dependency (due to varying in-plane lattice constants), and inevitably ultrahighresolution lithographic patterning (e.g., EBL, deep UV, soft-Xray) is needed.

Here, we present a versatile, wafer-scale framework for producing highly efficient, narrowband and customizable transmissive MSFAs based on grayscale (analog) lithography using a single lithographic processing step. We use grayscale lithography to generate spatially variant 3D MIM cavitiescovering the entirety of the pixel area-through usercontrolled, dose-modulated exposure schemes both in grayscale EBL (G-EBL) as proof-of-concept and in grayscale mask-based photolithography (G-PL) to demonstrate scalability to practical volume applications. The molecular weight of the resist is modified through exposure dose, thus making the rate of development a function of dose. For a grayscale dose profile, the remaining resist thickness (postdevelopment) depends on the dose and development time. Utilizing the 3D profile of the resist as the insulator material (cavity) in a MIM optical filter system, we fabricate spatially dependent 3D MIM structures as MSFAs with high transmission efficiency and narrow fwhm across the visible and near-IR. 


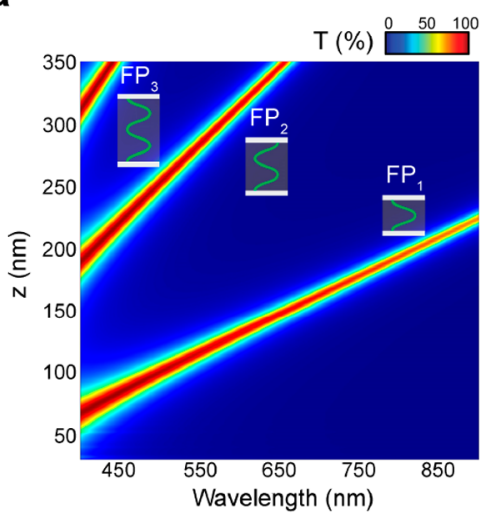

b

(i) (ii)

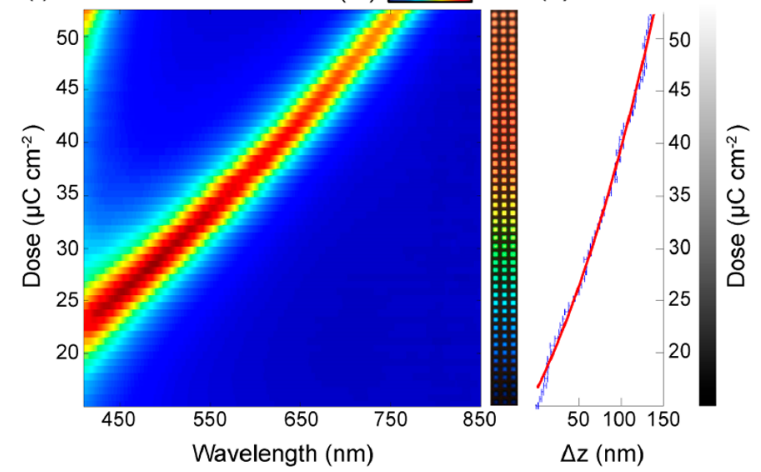

C

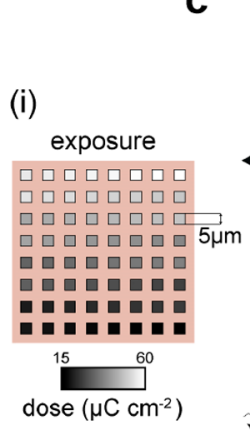

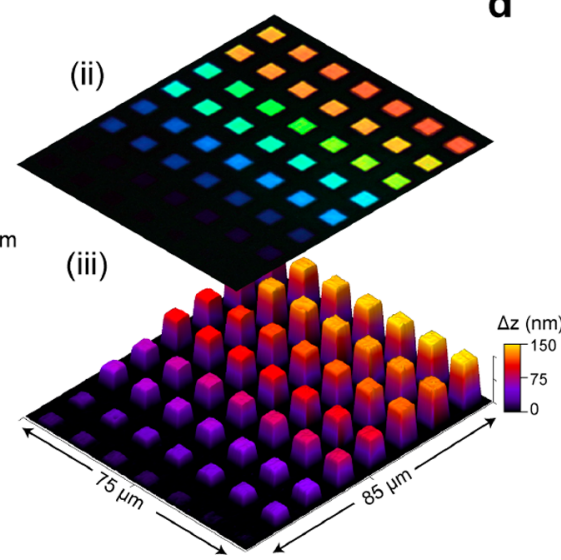

d

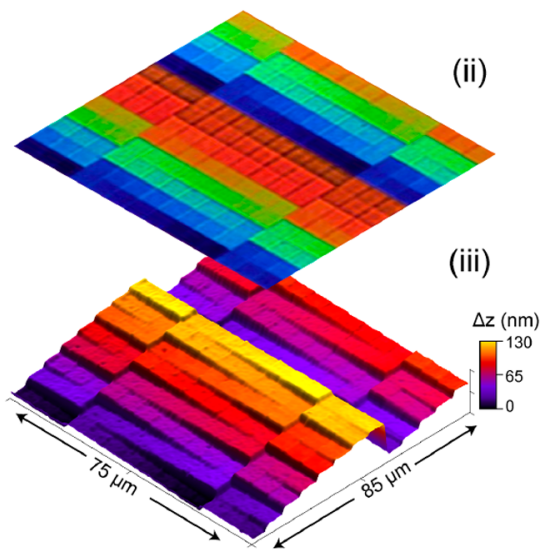

(i)

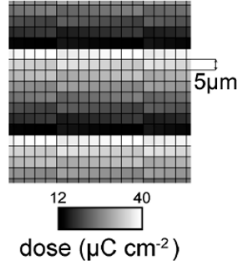

Figure 2. Grayscale exposure dose to color: experimental verification. (a) Finite-difference time-domain (FDTD) simulations of the optical transmission from a continuous Ag-based MIM cavity as a function of varying insulator thickness, with geometry: $\mathrm{SiO}_{2}(\mathrm{bulk})-\mathrm{Ag}(26 \mathrm{~nm})-\mathrm{resist}$ $(n=1.653)-\mathrm{Ag}(26 \mathrm{~nm})-\mathrm{MgF}_{2}(12 \mathrm{~nm})$. (b) Experimental demonstration of grayscale-to-dose pattern with the same layers as in (a): (i) Transmission spectra from dose-modulated $5 \mu \mathrm{m} \times 5 \mu \mathrm{m}$ squares (optical micrograph shown in inset), which results in increasing thickness and hence varying peak wavelengths; (ii) measured curve, using an AFM, linking dose and thickness (standard deviation error bars in blue, with overlaid polynomial-fitted red line). Only the first-order resonance is present at low doses, but for higher doses $\left(>50 \mu \mathrm{C} \mathrm{cm}^{-2}\right)$, the second-order mode is also excited. (c) Dose-modulated $5 \mu \mathrm{m} \times 5 \mu \mathrm{m}$ pixel array with $10 \mu \mathrm{m}$ spacing: (i) dose-modulated pattern, (ii) optical micrograph, and (iii) corresponding AFM data. (d) Same as (c) but with zero dead space.

\section{RESULTS AND DISCUSSION}

Our approach to the generation of visible color (spectral filtering) from grayscale dose modulation is depicted schematically in Figure 1. Custom MSFAs (Figure 1a) were created using dose-modulated exposure schemes to control local solubility of the resist (Figure 1b). To achieve this, the "resist sensitivity" was characterized so that a grayscale dose pattern could be applied to produce a physical three-dimensional resist profile. During resist development, different filter thicknesses, and hence wavelength selectivity, could be determined on a per-pixel basis by the total energy delivered to the resist volume.

We first performed electromagnetic simulations ${ }^{38}$ of the transmission response of a continuous MIM cavity with nondispersive insulator (resist; $n \approx 1.65)$ separating the $\mathrm{Ag}(26$ $\mathrm{nm}$ ) mirrors, with addition of a $12 \mathrm{~nm} \mathrm{MgF}_{2}$ encapsulation layer (Figure 2a, SI Section S1). These thicknesses were determined as a trade-off between transmission efficiency and fwhm (SI Figures S1 and S2). As the insulator thickness $(z)$ increases, the optical path length increases and the spectral position of the mode red-shifts accordingly. Moreover, multiple transmission peaks are excited for thicker insulator layers corresponding to the additional higher-order FP-type modes of the system.

To initially validate the grayscale-to-color approach, MIMbased MSFAs were fabricated using EBL with negative-tone MaN-2400 series photoresist (see Methods). An array of $5 \mu \mathrm{m}$ $(x-y$ dimensions) square pixels were assigned increasing dose values so that post G-EBL (with development time kept constant) each pixel had a different final thickness $(z)$. The layers of the final MIM structure consisted of an e-beam resist with two $26 \mathrm{~nm} \mathrm{Ag}$ mirrors and a $12 \mathrm{~nm} \mathrm{MgF}$ encapsulation layer. Experimental optical transmission spectra were recorded

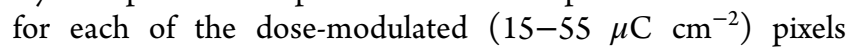
(Figure $2 \mathrm{~b}(\mathrm{i})$ ), with final thickness values (Figure 2b (ii)) confirmed using an atomic force microscope (AFM). The resultant transmission mode for each pixel spectrally red-shifts from $400 \mathrm{~nm}$ to $750 \mathrm{~nm}$ as the exposure dose increases, due to the thicker insulator layer, comparing favorably with simulation. The optimization of processing parameters for fabrication is detailed in SI Section S2 and SI Figures S3-S5. Transmission of up to $\sim 75 \%$ and relatively narrow full widths at half maximum (fwhm's) of $\sim 50 \mathrm{~nm}$ are observed in Figure $2 \mathrm{~b}$ (i), with $\Delta z$ thickness values up to $\sim 150 \mathrm{~nm}$ (Figure $2 \mathrm{~b}$, (ii)), in agreement with the simulation results (Figure 2a and 


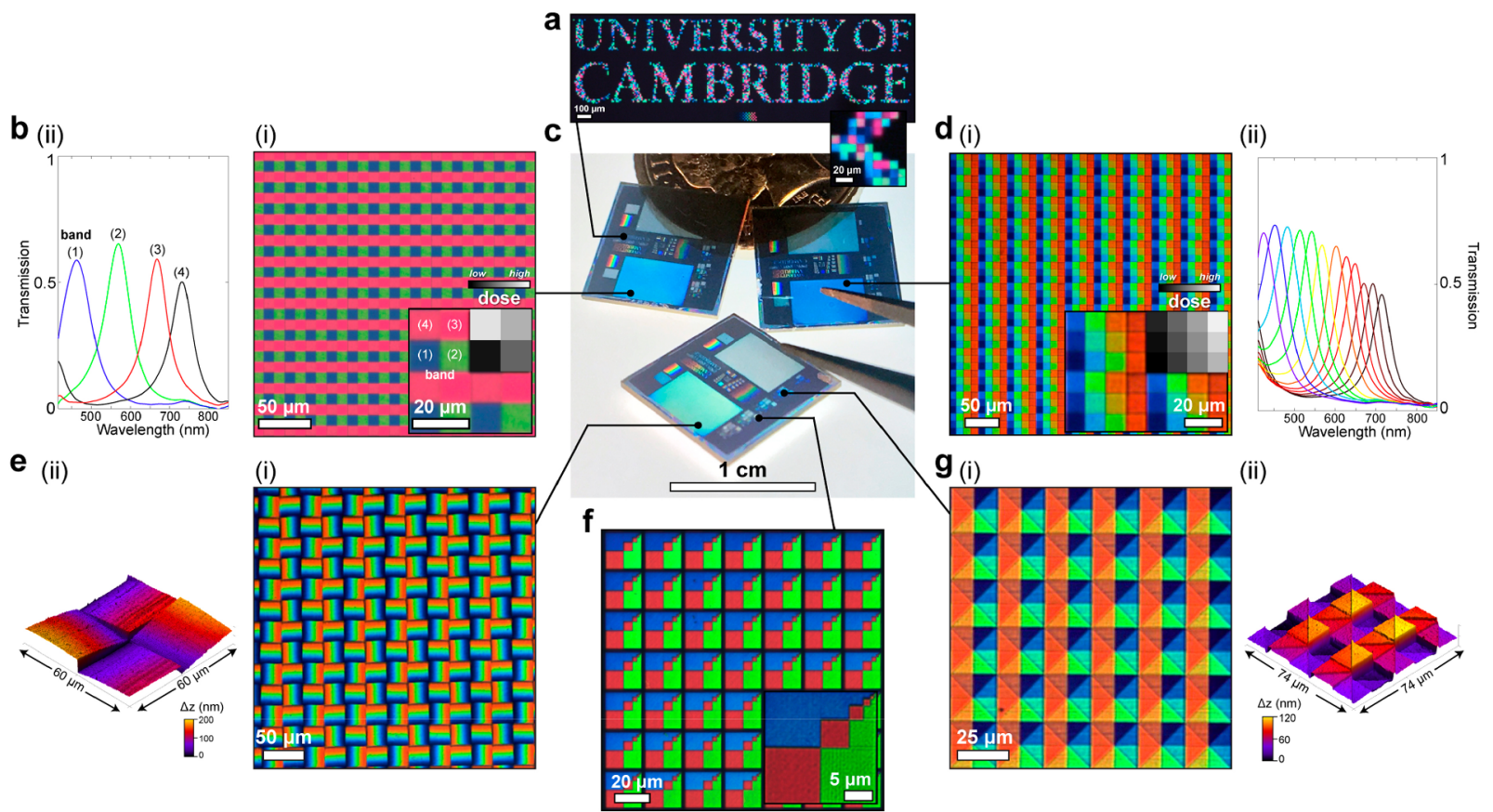

Figure 3. Demonstration of the versatility of grayscale MSFAs through patterned design variety. (a) Optical micrograph (with magnified inset) of the University of Cambridge logo text composed of $10 \mu \mathrm{m}$ pixels with a randomized exposure dose profile, hence random colors in transmission. (b) RGB+NIR MSFA (bands labeled in inset) with (i) optical micrograph in transmission and (ii) respective transmission spectra of the wavelength bands. (c) Photograph of three identically processed chips with a range of patterned designs on each chip with varying complexity. Each chip is processed in a single lithographic step in G-EBL. (d) Spectrally "ordered" $4 \times 3$ mosaic: (i) optical micrograph; (ii) transmission spectra. (e) $25 \mu \mathrm{m}$ linearly variable filter pixel design: (i) optical micrograph; (ii) AFM micrograph of the unit cell showing the in-plane height variation. (f) Optical micrograph (with magnified inset) of an array of RGB pixels with exponentially $\left(2^{-n}\right)$ decreasing pixel width, starting from $10 \mu \mathrm{m}$. (g) 25 $\mu \mathrm{m}$ discrete spiral phase pixel design: (i) optical micrograph; (ii) AFM micrograph. Transmission spectra represent averages of five different acquisitions, taken at random positions across the array.

SI Figure S1), demonstrating competitive spectral response characteristics.

Two different dose-modulated MIM arrays were then fabricated, clearly achieving varying colors as a result of variations in the cavity height. For isolated (Figure 2c) and dense (Figure $2 \mathrm{~d}$ ) pixel arrays, the EBL proximity effect ${ }^{39}$ leads to variation in the final thickness values and hence spectral response for an identical dose range. We therefore determined an empirical correction (decrease) to the dose range in order to achieve the desired spectral response for dense pixel arrays (see SI Section S2 and Figures S6 and S7). For our relatively thin $(\lesssim 200 \mathrm{~nm})$ MIM-based MSFA filters, the angular dependency is lower than typical multilayer alternating index filters. For larger chief ray angles of up to $30^{\circ}$, which are typical in smartphone-based image sensors, simulations show a small peak wavelength position shift $(\Delta \lambda)$ of $\sim 12 \mathrm{~nm}$ and $\sim 25 \mathrm{~nm}$ for transverse magnetic (TM) and transverse electric (TE) polarized input light, respectively (SI Figure S8).

To demonstrate the versatility of our MIM-based grayscale color approach, we next fabricated a variety of spatially varying optical filter designs, including several different MSFAs, on the same glass chip (Figure 3). Due to the high-sensitivity and high-resolution negative tone e-beam resist, the patterning exposure is relatively fast $\left(\sim\right.$ several $\left.\mathrm{mm}^{2} \mathrm{~min}^{-1}\right)$. The prototype MSFAs include two designs at $10 \mu \mathrm{m}$ pixel pitch: a $2 \times 2$ (4-band) RGB+NIR array (Figure $3 b$ ) and an ordered $4 \times 3$-band array (Figure $3 \mathrm{~d}$ ). Each array has total dimensions encompassing $2 \mathrm{~mm} \times 4 \mathrm{~mm}$, slightly larger than the active area of the image sensors used for imaging (SI Section S2). From a fabrication standpoint, the only difference between the two arrays is the exposure dose of each pixel. MSFAs across the UV-visible-NIR can be easily fabricated, as well as higherorder mosaic designs, linear filter arrays to unusual pyramidal structures, and spiral phase plates (see Figure $3 \mathrm{~d}-\mathrm{g}$, SI Figure S9). We have also verified G-EBL fabrication reproducibility (SI Figures S10 and S11) and pixels with lateral dimensions down to $460 \mathrm{~nm}$ (SI Figures S12 and S13), showing the approach yields consistent optical performance and is scalable to very small pixel sizes suitable for modern CMOS image sensors. For conventional commercial techniques to achieve similar design variety and complexity on a single chip with the high optical performance shown here would require many lithographic steps, materials, and masks, which is very process intensive and thus both expensive and time-consuming. Our MIM-based grayscale approach is therefore an attractive prospect for fabrication of complex, bespoke arrays.

Following process optimization (see Methods), we then exploited our MIM-based MSFAs for snapshot multispectral imaging. MSFAs containing a Bayer filter $2 \times 2$ (3-band, RGB) and a higher-order $3 \times 3$ (9-band) MSFA were fabricated on the same glass chip, with $11 \times 11 \mu$ m pixels. The $\mathrm{R}, \mathrm{G}$, and $\mathrm{B}$ bands of our Bayer MSFA filter (Figure 4a,b) have center wavelengths of 640,546 , and $427 \mathrm{~nm}( \pm 7 \mathrm{~nm})$; fwhm of 55, 66 , and $76 \mathrm{~nm}( \pm 7 \mathrm{~nm})$; and peak transmission efficiencies of $75 \%, 82 \%$, and $88 \%( \pm 6 \%)$, respectively.

The Bayer MSFA was then used to image a multispectral test scene (Macbeth ColorChecker with a Rubik's cube, Figure 4c,d) with imaging setup detailed in SI Figure S14. The filter pixels used are slightly larger than the sensor pixels to accommodate for the $\sim 1 \mathrm{~mm}$ distance from the CMOS image 
a

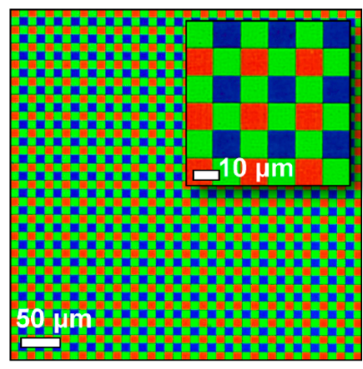

b

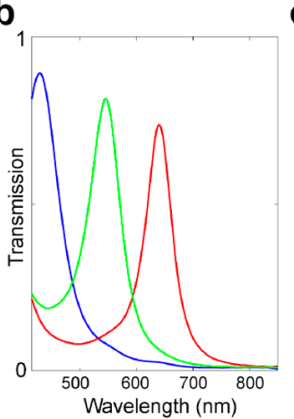

C

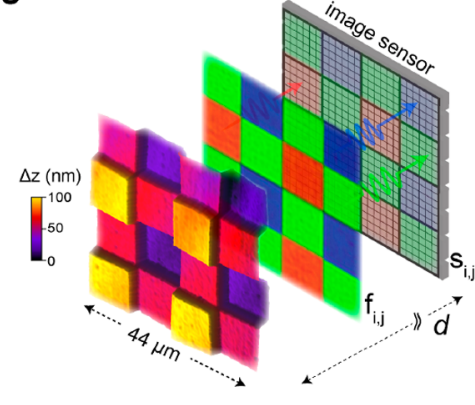

d

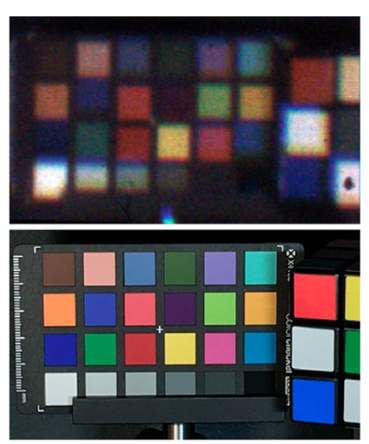

e

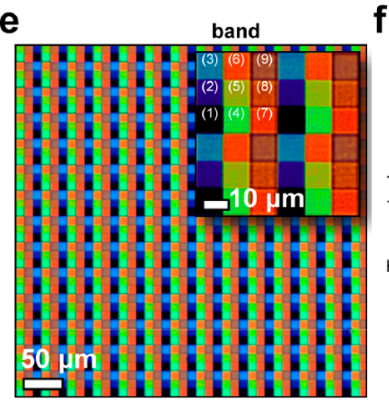

f

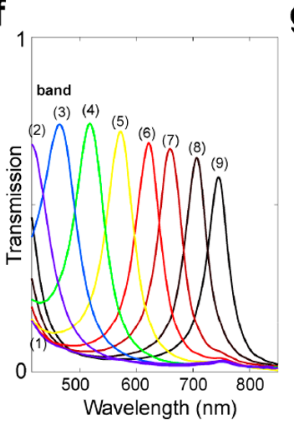

g

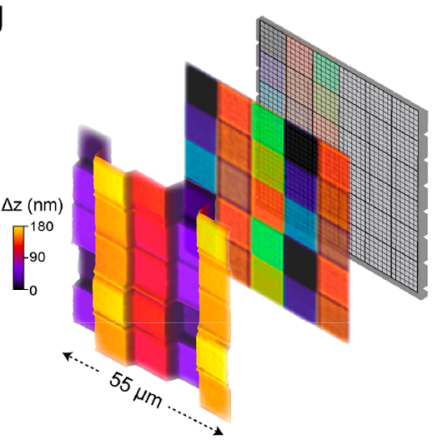

$\mathbf{h}$

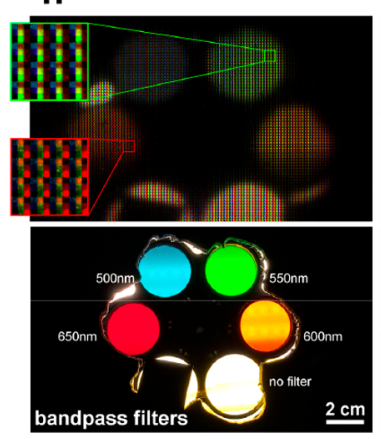

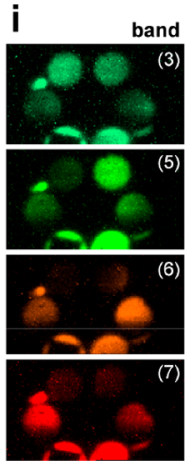

Figure 4. Multispectral imaging through a Bayer filter design and 9-band $(3 \times 3)$ MSFA. Bayer filter: (a) Optical micrograph of the mosaic with respective transmission spectra (b) of the 3 bands (RGB). (c) Imaging: Physical representation of the MSFA in front of the image sensor: experimental AFM micrograph, optical micrograph, and image sensor schematic, where $d$ is the distance of the MSFA from the sensor plane $(\sim 1$ $\mathrm{mm}$ ). The experimental imaging setup is shown in SI Figure S16. (d) A snapshot of the imaging test scene, including Macbeth ColorChecker chart and Rubik's cube, captured with a monochrome image sensor through our mosaic (top) and using a conventional smartphone (bottom), for reference. Aside from demosaicing, there is no postprocessing (enhancement) of the color in the image acquired through our mosaic. $3 \times 3$ MSFA: (e) Optical micrograph of the $3 \times 3$ mosaic with respective transmission spectra (f) of the 9 bands (labeled) in the MSFA. (g) Multispectral imaging: Schematic representation of the MSFA in front of the image sensor: experimental AFM micrograph, optical micrograph, and image sensor schematic, where $d$ is the distance of the MSFA from the sensor plane $(\sim 1 \mathrm{~mm})$. 2D intensity matrices from the monochrome image sensor (captured through the MSFA) with illumination from a supercontinuum source are shown in SI Section S 3.3 and SI Video S1 at four different center wavelengths: 500,550,600, $650 \mathrm{~nm}$; fwhm $10 \pm 2 \mathrm{~nm}$. (h) Multispectral test scene comprising a rear-illuminated filter wheel with four different bandpass filters. (Top) "Raw" color image captured using the monochrome image sensor through our MSFA with individual MSFA pixels visible. (Bottom) Reference image of test scene (comprising 4 bandpass filters across the visible spectrum) taken using a conventional smartphone image sensor. (i) Demosaiced color-coded images for four different wavelength bands obtained from the "raw" image in (h) (top), with labels denoting band.

sensor surface (Methods). With our Bayer filter positioned in front of the monochrome image sensor (Figure 4c) a raw image can be captured and demosaiced (SI Figure S15) to form a standard three-band RGB image (Figure 4d). Without enhancement (i.e., standard color correction techniques used in imaging) the captured raw image provides accurate reconstruction of the multispectral test scene when compared to a commercial RGB color camera.

We then characterized the custom $3 \times 3$ (9-band: 8-band + 1 reference band) MSFA (Figure 4e,f) and placed it in front of the image sensor (Figure $4 \mathrm{~g}$ ) to demonstrate snapshot multispectral imaging. From shortest to longest wavelength, the $3 \times 3$ (8-band) array has center wavelengths of 415, 463, $518,572,621,660,706$, and $725 \mathrm{~nm}( \pm 6 \mathrm{~nm})$; fwhm's of 81 , $75,66,58,55,53,48$, and $46 \mathrm{~nm}( \pm 7 \mathrm{~nm})$; and peak transmission efficiencies of $69 \%, 75 \%, 74 \%, 72 \%, 69 \%, 68 \%$, $65 \%$, and $59 \%( \pm 7 \%)$, respectively. Initially we used a supercontinuum white light laser source as a collimated input, recording the intensity response through the MSFA at $10 \mathrm{~nm}$ steps with center wavelengths in the range 450-750 nm (see SI Video S1 and SI Figure S16). Multispectral imaging was then performed using a dynamic test scene consisting of spatially separated optical bandpass filters backlit with a white LED floodlight (Figure 4h). The raw color image (Figure 4h, top) is composed of the monochrome intensity multiplied by the $3 \times 3$ (9-band) MSFA matrix, showing the power distribution across the nine bands. The demosaiced images for four bands of the MSFA (Figure 4i) show the ability to clearly discriminate the spectral information within the test scene.

On the basis of the realization of the grayscale-to-color custom MSFA concept using maskless EBL, we then translated it into mask-based G-PL to achieve wafer-level fabrication and illustrate the scalability of custom MSFA production to industrial processing. To achieve this, we represented a grayscale dose matrix photomask (Figure 5a) with a binary amplitude mask. Through lateral translations and adjustment of the flood exposure dose (Figure 5), the grayscale dose profile can then be simply delivered and easily translated to commercial wafer level processing standards. For our demonstration, we employed the binary mask approach, as the final optical performance from the two methods is identical.

SU-8 (2000 series) photoresist, widely used commercially as a negative-tone resist, is used for this scalability demonstration. 


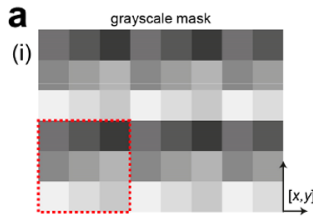

(ii)
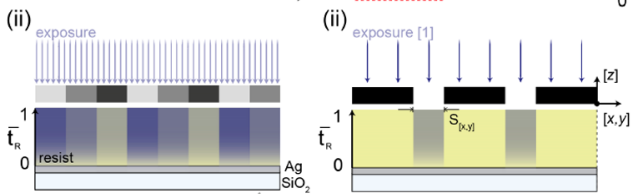

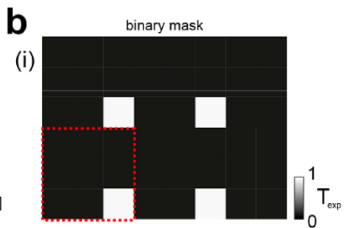

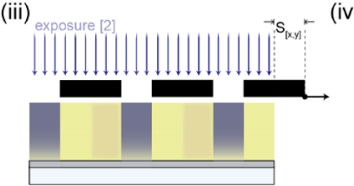

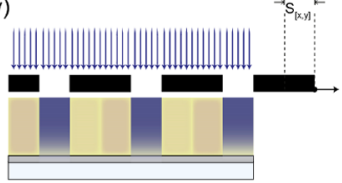

(v)
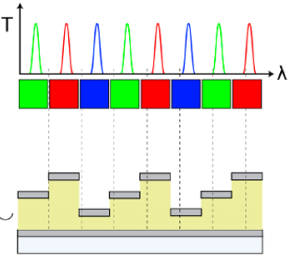

d

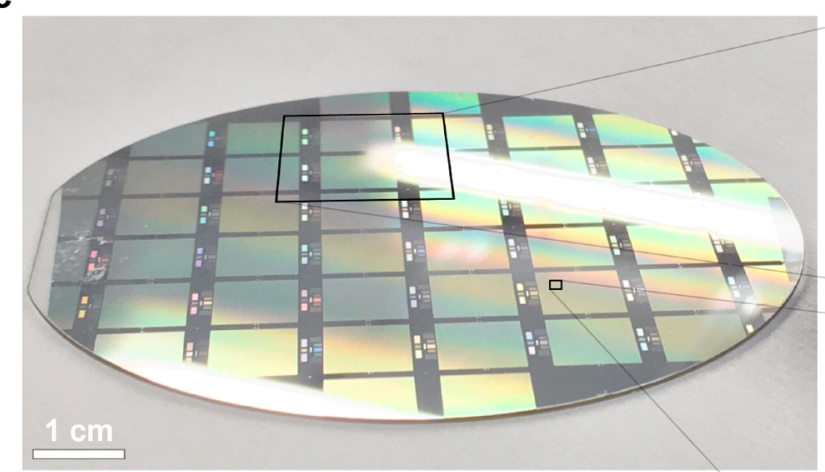

h

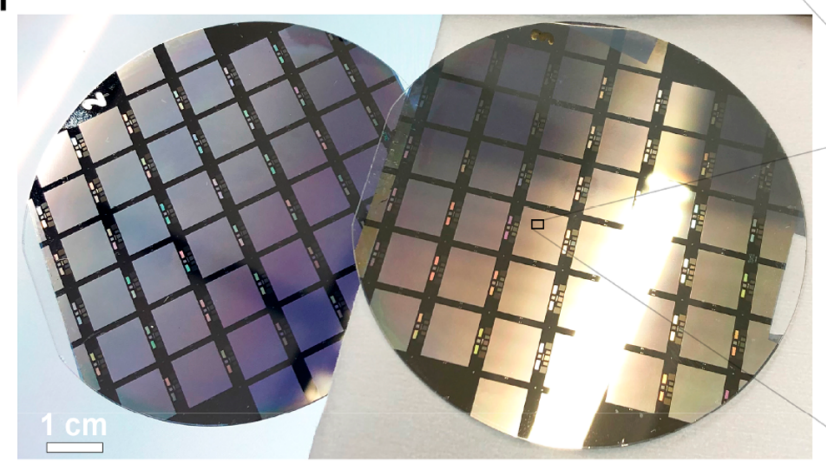

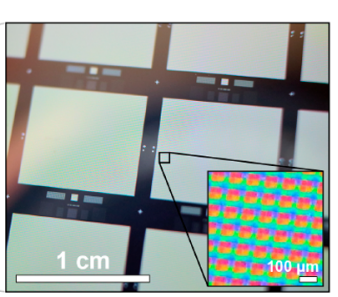
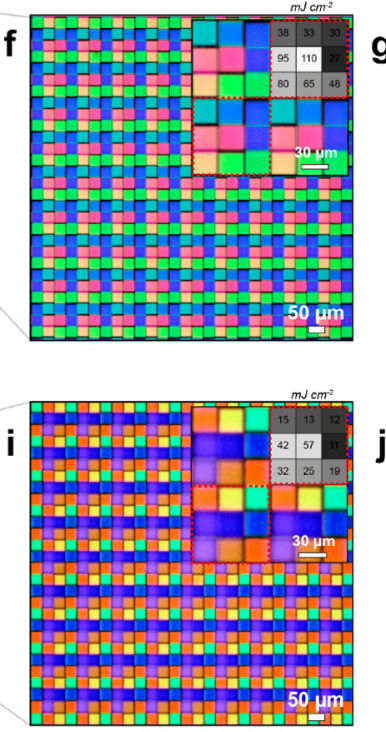

e

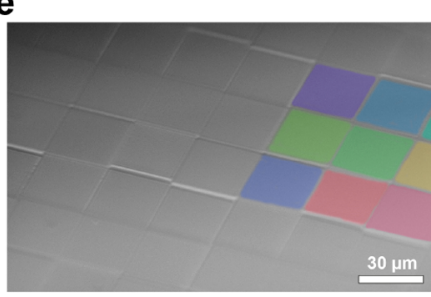

g
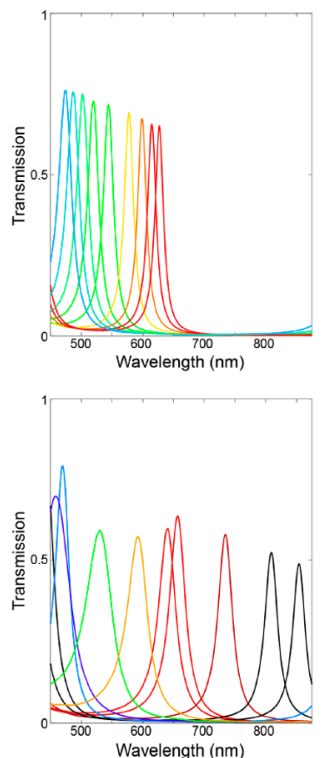

Figure 5. Wafer-scale grayscale-to-color MSFA fabrication. Photolithography-based MSFA fabrication process flow schematic comparing two proposed approaches: a grayscale photomask (a) or binary photomask (b); both result in equivalent MSFAs. (a) (i) $3 \times 3$ grayscale photomask: 9 levels of optical transmission, one per spectral band. A single flood exposure can be performed imparting a spatially varying dose profile into the photoresist (ii). (b) (i) $3 \times 3$ binary photomask: A single transparent pixel is repeated in a $3 \times 3$ array (MSFA unit cell). The mask is translated inplane for each spectral pixel, with varying exposure levels (ii-iv). Once processed, the spectral response of the final MSFA (v) is identical to that from (a). (c) Photograph of a 3 in. wafer with 32 9-band MSFAs (utilizing second-order resonances), with a zoomed-in region captured with a macro lens (d) and tiled SEM micrograph (e) of the same region. (f) Optical micrograph (transmission) of a different region of the wafer, with labeled equivalent exposure pattern (inset) and corresponding transmission spectra (g) for each spectral band. (h) Photograph of two 3 in. MSFA wafers (utilizing first- and second-order resonances), with optical micrograph of one MSFA (i) and its corresponding transmission spectra for each band $(\mathrm{j})$.

A 3 in. wafer was patterned with $\sim 32$ MSFAs each containing nine spectral bands $(3 \times 3)$ using the mask-based PL grayscaleto-color approach (Figure 5c, see Methods). The pixels in the $3 \times 3$ (9-band) mosaic are $30 \times 30 \mu \mathrm{m}$, and the layers of the final MIM structure consisted of SU-8 resist with two $38 \mathrm{~nm}$ $\mathrm{Ag}$ mirrors and a $38 \mathrm{~nm} \mathrm{SiO}_{2}$ encapsulation layer. The uniformity of adjacent MSFAs is highlighted using a DSLR camera and macro lens (Figure 5d) and a tilted SEM micrograph of several unit cells (Figure 5e, for further morphological inspection, see SI Figure S17). A different region of the same wafer is analyzed under the optical microscope (Figure 5f), with the inset showing a magnified region and overlaid equivalent exposure dose matrix, ranging from 27 to $110 \mathrm{~mJ} \mathrm{~cm}^{-2}$. The dose range and processing conditions have been empirically optimized (see SI Figures S18 and S19). The corresponding resultant transmission spectra (Figure 5g) span 460-630 nm with excellent optical characteristics, from shortest to longest wavelength: fwhm's of $27,26,24,22,21,20,19,18$, and $17 \mathrm{~nm}( \pm 5 \mathrm{~nm})$ and peak transmission efficiencies of $76 \%, 76 \%, 75 \%, 73 \%, 72 \%, 70 \%$, $68 \%, 66 \%$, and $65 \%( \pm 6 \%)$. This demonstration wafer, chosen from batches of multiple wafers (see SI Figure S20), exhibits 
the narrower second-order FP-type resonances (thicker final resist thickness), showing the versatility of our bespoke MSFA approach; by adjusting the flood exposure dose, we can easily incorporate first- and second-order modes, as shown in other demonstration wafers (Figure $5 \mathrm{~h}-\mathrm{j}$ ).

Our bespoke wafer-level MIM-based MSFAs are able to outperform alternative approaches for color filter fabrication, such as plasmonic and high-index dielectric nanostructure arrays/metasurfaces, in several ways. For example, in comparison to recent progress in spatially variant spectral filters, ${ }^{10,12,14-21}$ our MSFA transmission bands (Figure $5 \mathrm{~g}$ ) are narrower, have higher transmission efficiencies, exhibit no polarization dependency (up to high angle of incidence chief ray angles), and, most importantly, have been fabricated at the wafer level (over large areas), illustrating translation ease to commercial processing. The next step would be to replace the $\mathrm{Ag}$ mirrors with few-layer alternating index all-dielectric mirrors (e.g., $\left.\mathrm{TiO}_{2} / \mathrm{SiO}_{2}\right)^{11}$ - during the two physical vapor deposition processing steps-to enable a fully robust, chemically inert, and cost-effective approach. In addition, to further improve reproducibility, a grayscale $\mathrm{Cr}$ photomask can be utilized, whereby $\mathrm{Cr}$ thickness variation provides opacity modulation (SI Figure S24). The manufacture of a grayscale $\mathrm{Cr}$ mask is conceivable through repeated binary-step reactive ion etching or wet etching steps, one for each spectral band. Once manufactured, a single flood exposure would provide the required grayscale exposure dose. Such a grayscale mask would minimize misalignment issues, hence improving reproducibility and increasing throughput.

\section{CONCLUSION}

In summary, we have demonstrated fabrication of highefficiency, narrowband, highly customizable MSFAs with full pixel coverage, using a simplified single lithographic processing step (grayscale-to-color) and demonstrated that it is scalable to wafer-level fabrication for practical application. A grayscale dose matrix is utilized to generate customizable insulator thickness profiles in MIM geometries, producing optical filters spanning the UV-visible-NIR. We first proved the concept (at the low volume scale) using grayscale maskless e-beam lithography by fabricating a diverse range of MSFA devices with varying mosaic complexities; imaging performance was demonstrated by placing these fabricated MSFAs in front of a commercial image sensor and performing pixel-wise discrimination of different wavelength bands in a multispectral test scene. We then used mask-based UV grayscale photolithography for wafer-level fabrication of MSFAs, maintaining high transmission efficiency $(\sim 75 \%)$ and narrow line widths ( 25 nm). Current manufacturing methods for producing spatially variant optical filters, from linearly variable filter arrays to MSFAs, will typically use $N$-lithographic steps for $N$ wavelength bands and require a variety of materials, ${ }^{9,40}$ limiting their customizability. By contrast, this versatile approach requires only a single lithographic processing step and the same materials for each band, making it highly customizable. Unlike other reported single-step approaches with a single material layer, such as nanostructured filters (plasmonic, alldielectric, or otherwise), ${ }^{12,22,23}$ it does not require expensive ultra-high-resolution subpixel patterning (e.g., EBL, deep-UV, soft-X-ray), hence is compatible with the resolution limit of widespread, industrially standard UV lithography.

The combination of high optical performance, customizability, and fabrication at the wafer level using UV photolithography surpasses the current state-of-the-art for producing MSFAs. ${ }^{10,12,14-23}$ Our wafer-level approach to MIM-based MSFAs could therefore enable a whole new range of custom multispectral image sensors targeted to different applications. Furthermore, we envisage the wafer-level methodology reported here could play a significant role in reducing the fabrication complexity and cost of intricate optical filter devices for high-volume MSFAs such as the Bayer filter. This MSFA fabrication framework could be a key enabling step toward widespread industrial adoption of multispectral image sensors.

\section{METHODS}

Fabrication Techniques. EBL Processing. MaN-2400 series negative tone photoresist (Micro Resist Technology $\mathrm{GmbH}$ ) is utilized for the low-volume (proof of concept) part of this study, due to its high-resolution capability for EBL in combination with relatively high sensitivity. Double-sided polished borosilicate (Borofloat 33) glass (Pi-kem), thickness $500 \pm 25 \mu \mathrm{m}$, is diced into $1 \mathrm{~cm}^{2}$ samples. The glass samples are cleaned in successive ultrasonic baths of acetone (Fisher Scientific) and isopropyl alcohol (IPA) (Fisher Scientific) for $10 \mathrm{~min}$, blow-dried with ultra-high-purity (UHP) compressed $\mathrm{N}_{2}$, and dehydrated at $200{ }^{\circ} \mathrm{C}$ for $10 \mathrm{~min}$.

A $1.5 \mathrm{~nm} \mathrm{Ti}$ adhesion layer is thermally evaporated (Edwards E306 evaporator) (base pressure $\sim 2 \times 10^{-6} \mathrm{mbar}$, deposition at $0.1 \mathrm{~nm} \mathrm{~s}^{-1}$ ), followed by a $26 \mathrm{~nm}$ layer of $\mathrm{Ag}$ (with relatively fast deposition, $0.2-0.3 \mathrm{~nm} \mathrm{~s}^{-1}$, for improved optical performance), followed by a second $1.5 \mathrm{~nm}$ Ti layer. The first Ti layer promotes adhesion between the glass and Ag, and the second increases the wettability of $\mathrm{Ag}$ for resist spincoating and increases chemical stability by reducing $\mathrm{Ag}$ oxidation. The optimal thickness of the $\mathrm{Ag}$ is determined through simulations (SI Figure S1), trading transmittance for fwhm. The thickness of the $\mathrm{Ti}$ layers is such that resist wettability is increased and adhesion is promoted with minimal effect on optical transmittance. MaN-2405 eB resist is spincoated on top of the samples at $5200 \mathrm{rpm}$ for $45 \mathrm{~s}$ to form a $\sim 350 \mathrm{~nm}$ layer, then baked at $90^{\circ} \mathrm{C}$ for $3 \mathrm{~min}$. High-voltage $(80 \mathrm{kV})$, high-current $(4.2 \mathrm{nA})$ EBL (nB1, Nanobeam Ltd..) is used for the patterning. The bottom metallic mirror layer additionally acts to dissipate accumulated charge during electron beam exposure. The MSFAs have total area dimensions $\sim 1.1 \times$ greater than the image sensor area $(4.85$ $\mathrm{mm}$ diagonal) to correct for the proximity effect (SI Figure S8) and ensure all sensor pixels are utilized. The effect of stitching error is reduced due to the rectangular geometry (edges) of the patterns corresponding to the main-field and subfield fractures. No sample registration marks are used for the samples shown in this study. The high current, in combination with low critical dose (due to inherent high sensitivity) of the resist, allows for fabrication over relatively large areas $(\sim$ millimeters $)$ in quick time periods. For example, the large MSFA $(\sim 2 \times 4 \mathrm{~mm})$ in Figure $3 \mathrm{c}$ required a total exposure time of $\sim 20 \mathrm{~min}$. The critical parameters in this grayscale-to-color study are the exposure dose and development conditions, which are determined empirically through a variety of dose tests (SI Section $S$ 2.1). For this study, a dose range of $5-75 \mu \mathrm{C} \mathrm{cm}^{-2}$ is used and full concentration AZ-726-MIF (AZ Electronic Materials) developer solution for $\sim 10 \mathrm{~s}$, followed by two deionized (DI) water (stopper) rinses for $4 \mathrm{~min}$ and UHP compressed $\mathrm{N}_{2}$ blow dry. A postdevelopment bake $\left(90{ }^{\circ} \mathrm{C}\right.$ for $30 \mathrm{~s})$-in which the resist is brought within close proximity to 
its glass transition temperature-is subsequently performed, which yields a smoother surface before the second mirror deposition and improves optical performance (SI Section S 2.3 and Figure S21). The top metal, a $26 \mathrm{~nm}$ layer of $\mathrm{Ag}$, is thermally evaporated (deposition at $0.2-0.3 \mathrm{~nm} \mathrm{~s}^{-1}$ ) followed by a $12 \mathrm{~nm}$ layer of $\mathrm{MgF}_{2}$. This final encapsulation layer adds chemical and mechanical stability to the MSFAs with minimal, if not improved, effect on optical properties (SI Figure S2).

PL Processing. SU-8 2000 series negative photoresist (Microchem) is utilized for the wafer-level MSFA processing part of this study. It is widely used commercially, has high thermal stability (glass transition temperature $>200^{\circ} \mathrm{C}$ ), and is designed to be permanent; it is typically incorporated into the final processed device. A SUSS Microtec MA/BA6 semiautomated mask aligner, with $365 \mathrm{~nm}$ (i-line) exposure and $5 \times$ alignment objectives, was operated in hard contact mode. The 3 in. double-sided polished borosilicate (Borofloat 33) glass wafers (Pi-kem), thickness $500 \pm 25 \mu \mathrm{m}$, are cleaned in successive ultrasonic baths of acetone and IPA for $10 \mathrm{~min}$, rinsed in DI water, blow-dried with UHP compressed $\mathrm{N}_{2}$, and dehydrated at $200{ }^{\circ} \mathrm{C}$ for $10 \mathrm{~min}$.

A set of crosshair alignment markers $(30 \times 30 \mu \mathrm{m})$ are patterned with PL (500 mJ cm ${ }^{-2}$ exposure) using MaN-1400 series photoresist $\left(2500 \mathrm{rpm}, 50 \mathrm{~s}\right.$; soft-bake: $95^{\circ} \mathrm{C}, 2 \mathrm{~min}$ ) and developed with AZ $726 \mathrm{MIF}$ for $3 \mathrm{~min}$. The first metallic mirror, composed of $\mathrm{Ti} / \mathrm{Ag} / \mathrm{SiO}_{2}(1.2 / 38 / 12 \mathrm{~nm})$, is deposited on the marker-patterned glass using a Lesker PVD-75 electron beam evaporator (base pressure $\sim 9 \times 10^{-6}$ mbar, deposition rate $0.5 \AA \mathrm{s}^{-1}$ ). During the deposition the wafer chuck is rotated at $\sim 5 \mathrm{rpm}$ in order to increase coverage uniformity. Lift-off is performed in an ultrasonic bath of $N$ methyl-2-pyrrolidone (NMP) at $60{ }^{\circ} \mathrm{C}$ for $3 \mathrm{~min}$, followed by wafer cleaning (acetone, IPA, DI rinse, $\mathrm{N}_{2}$ dry, dehydration bake). The resultant wafers have a continuous bottom metallic mirror with a regular array of transparent alignment markers.

SU-8 2000.5 photoresist is spin-coated on top of the wafers at $5500 \mathrm{rpm}$ for $50 \mathrm{~s}$ to form a $\sim 350 \mathrm{~nm}$ layer, then soft-baked at $95{ }^{\circ} \mathrm{C}$ for $3 \mathrm{~min}$. The binary amplitude $\mathrm{Cr}$ photomask (JD Photodata) consists of a repeating array of transparent square pixels, separated by the unit cell size. For the $30 \mu \mathrm{m}$ pixels in this work, the photomask pixels are separated in order to give a final $3 \times 3$ (9-band) mosaic, hence $120 \times 120 \mu \mathrm{m}$ separation. The mask aligner is operated in constant dose and hard contact mode. The mask (with a $3 \times 3$ array of alignment crosshairs) is translated above the substrate (aligned with each band in the 3 $\times 3$ array) and flood exposed; the dose matrix ranges from 10 to $120 \mathrm{~mJ} \mathrm{~cm}^{-2}$. The exposure dose profile to yield the required insulator thickness for spectral filtering (visible) in the MIM stack has been empirically optimized. A postexposure bake of $65{ }^{\circ} \mathrm{C}$ for $2 \mathrm{~min}$ is then followed by a $2 \mathrm{~min}$ development in 1-methoxy-2-propanol acetate (PGMEA), IPA rinse, and $\mathrm{N}_{2}$ blow dry. The resultant structure is a bottom metallic mirror with a $3 \mathrm{D}$ thickness profile (cavities) across the entire wafer. The second metallic mirror, composed of $\mathrm{Ag} /$ $\mathrm{SiO}_{2}(38 / 38 \mathrm{~nm})$, is deposited using the electron beam evaporator. Custom horizontal and vertical alignment markers are patterned (SI Figure S21b) in order to determine the alignment accuracy of the final MSFA pixels.

Optical and Morphological Characterizations. Surface morphology is characterized using an AFM (Asylum Research MFP-3D) in conjunction with Al-reflex-coated $\mathrm{Si}$ probes (Budget Sensors, Sigma-Aldrich) operated primarily in tapping mode. Scan speed, voltage set-point, and drive amplitude are modified dependent on the feature morphology. Gwyddion software is used for the AFM data visualization and analysis. The raw surface data are plane leveled, scars (strokes) and noise minimized, and the resultant data are presented in 3D topography form. The average height (and standard deviation) of each pixel (such as in Figure $2 \mathrm{~b}$ (ii)) is obtained using the in-built statistical analysis toolbox. A LEO Gemini 1530VP field emission scanning electron microscope (SEM) operating at $1-5 \mathrm{keV}$ is used for imaging the surface of samples (in-lens operation), which are fixed on angled SEM stubs for nonnormal incidence imaging. Carl Zeiss software (SmartSEM) is used to control the SEM and obtain images at several magnifications. The optical characterization is performed using a modified Olympus BX-51 polarizing optical microscope (halogen light source with IR filters removed) attached via a $300 \mu \mathrm{m}$ core multimode optical fiber (Ocean Optics OP400-2SR MMF) to a UV-visible spectrometer (Ocean Optics HR2000+) and second optical arm to a digital camera (Lumenera Infinity-2 2MP CCD) for surface imaging (SI Figure S22). The spectra are normalized to transmission through equivalent thickness borosilicate glass (bright state) and no input light (dark state) using Ocean Optics OceanView software.

Further characterization is performed using a supercontinuum white light source and tunable filter (NKT Photonics: SuperK COMPACT source and SuperK VARIA filter). SI Video S1 shows the normalized intensity map of the image sensor as a function of increasing input wavelength (450-750 $\mathrm{nm} ; 10 \mathrm{~nm}$ line width), generated with a supercontinuum white light laser source. The laser is fiber coupled, expanded, and collimated to be used as an input source to the MSFA sensor. There are no imaging optics in the system. The video shows (right) how the intensity response changes as a function of input wavelength, with the respective MSFA (left).

For the imaging experiments, the test scene is composed of a Macbeth ColorChecker chart (A5 size) along with a Rubik's cube, which is imaged via a series of lenses through the custom MSFAs onto a CMOS image sensor (SI Figure S14). A USB 3.0 monochrome 2MP Basler daA1920-30 $\mu \mathrm{m}$ area-scan camera is used (Aptina MT9P031 CMOS image sensor), with a total sensor size of $4.2 \mathrm{~mm} \times 2.4 \mathrm{~mm}$, resolution of $1920 \times 1080$, and $2.2 \mu \mathrm{m} \times 2.2 \mu \mathrm{m}$ pixel size. Each filter pixel has dimensions of $11 \mu \mathrm{m} \times 11 \mu \mathrm{m}$, corresponding to a $5 \times 5$ array of the image sensor pixels. At an image sensor resolution of $1920 \times 1080$ the $1: 5$ trade-off in spatial resolution means the effective resolution of our images is $384 \times 216$.

The image sensor is mounted at the end of a custom optical cage system using a 3D printed (Ultimaker 2+) mount. An inhouse-built XYZ translation mount holds the MSFAs, which are fabricated on $10 \mathrm{~mm} \times 10 \mathrm{~mm}$ borosilicate glass chips. The imaging optics consist of three achromatic AR-coated lenses (Thorlabs LSBO8-A series): the first (a concave lens) demagnifies the scene, the second collimates this virtual image (placed at the focal point of the first lens), and the third focuses the light onto the image sensor, through the MSFA mounted in front of it. An aperture stop is located after the third lens, limiting the range of ray angle impingement on the MSFA and thus onto the image sensor. The MSFAs, fixed in a custom 3D printed mount, are brought close to the borosilicate cover glass (thickness $0.4 \mathrm{~mm}$ ) of the image sensor. Using the image sensor manual (Micron MT9P031 manual and Basler AW001305 documents) to determine the physical sensor geometry, the minimum distance of the MSFA from the image 
sensor die (plane) is estimated at $\sim 0.525 \pm 0.05 \mathrm{~mm}$. The MSFA is translated in XYZ in order to align the pixels of the filter array with the pixels of the image sensor. For the MSFA imaging results, a series of optical bandpass filters (Thorlabs FKB-VIS-10 series; $10 \mathrm{~nm}$ fwhm) are utilized in a filter wheel mount, backlit with a $50 \mathrm{~W}$ white light $(4000 \mathrm{~K})$ floodlight LED array. The reflected light from the object test scene is imaged through the MSFA onto a monochrome image sensor. The process of starting with the raw 2D intensity matrix (from the image sensor), which has no wavelength-specific information, and determining the wavelength-specific information ( $N$-band $\times 2 \mathrm{D}$-image data) is performed using a custom code in MATLAB (SI Figure S15).

Numerical Simulations. A commercial-grade simulator (Lumerical FDTD solutions) based on the finite-difference time-domain (FDTD) method was used to perform the calculations. ${ }^{38}$ MIM stacks are simulated using a dielectric between two metal layers ( $z$-dimension). Periodic boundary conditions are used $(x-y$ boundaries of the unit cell) and perfectly matched layers ( $z$-boundary) along the direction of propagation. A uniform 2D mesh (Yee-cell) with dimensions of $\leq 1 \mathrm{~nm}$ and broadband-pulse plane-wave (350-1000 nm) injection source at a significant distance (several micrometers) above the sample are used. For the $E$ - and $H$-field intensity plots, an additional finer mesh is included, whereby the smallest cubic mesh size is $<0.01 \mathrm{~nm}$ ( $z$-direction). Complex dispersive material models are used for $\mathrm{Ag}$ (Johnson and Christy model) and $\mathrm{SiO}_{2}$ (material data), whereas a real-only refractive index of 1.65 is used for MaN-2400 series photoresist (Microchem: Material data sheet) and 1.4 for the transparent $\mathrm{MgF}_{2}$ capping layer. Transmittance and reflectance values are calculated from $1 \mathrm{D}$ power monitors positioned above the range of structures and source injection.

\section{ASSOCIATED CONTENT}

\section{S Supporting Information}

The Supporting Information is available free of charge on the ACS Publications website at DOI: 10.1021/acsphotonics.9b01196.

Simulations of transmission and angle dependency; fabrication process optimization and materials considerations (PDF)

Movie illustrating MSFA characterization (AVI)

\section{AUTHOR INFORMATION}

\section{Corresponding Authors}

*E-mail: cw507@cam.ac.uk.

*E-mail: seb53@cam.ac.uk.

\section{ORCID $\odot$}

Calum Williams: 0000-0002-6432-6515

Sarah E. Bohndiek: 0000-0003-0371-8635

\section{Author Contributions}

C.W. conceived the idea. C.W. carried out the design and simulation of the devices. C.W. fabricated the devices and performed the experimental measurements. C.W. characterized the devices. G.S.D.G., T.D.W., and S.E.B. supervised the study. C.W., G.S.D.G., T.D.W., and S.E.B. analyzed the data and wrote the manuscript.

\section{Notes}

The authors declare no competing financial interest.
Please note that the dataset to accompany this manuscript will be posted at: https://doi.org/10.17863/CAM.45120 before publication.

\section{ACKNOWLEDGMENTS}

This work was supported by Cancer Research UK (C55962/ A24669, C47594/A21102, C14303/A17197, C47594/ A16267) and the Engineering and Physical Sciences Research Council (EP/R003599/1). C.W. would like to acknowledge the support of Wolfson College, Cambridge. The authors would like to acknowledge the following researchers for their fruitful discussions and help with the work: Sophia Gruber, Catherine Fitzpatrick, Girish Rughoobur, Dale Waterhouse, Jonghee Yoon, Siri Luthman, and Christian Kuppe.

\section{REFERENCES}

(1) Kuroda, T. Essential Principles of Image Sensors; CRC Press, 2017.

(2) Lu, G.; Fei, B. Medical Hyperspectral Imaging: A Review. J. Biomed. Opt. 2014, 19 (1), No. 010901.

(3) Lapray, P. J.; Wang, X.; Thomas, J. B.; Gouton, P. Multispectral Filter Arrays: Recent Advances and Practical Implementation. Sensors 2014, 14 (11), 21626-21659.

(4) Bayer, B. E. Color Imaging Array. US3971065A, 1975.

(5) Geelen, B.; Tack, N.; Lambrechts, A. A Compact Snapshot Multispectral Imager with a Monolithically Integrated Per-Pixel Filter Mosaic. Proc. SPIE 2014, 8974, 89740L.

(6) Hagen, N.; Kudenov, M. W. Review of Snapshot Spectral Imaging Technologies. Opt. Eng. 2013, 52 (9), 090901.

(7) Coffey, V. C. Multispectral Imaging Moves into the Mainstream. Opt. Photonics News 2012, 23 (4), 18-24.

(8) Carmo, J. P.; Rocha, R. P.; Bartek, M.; De Graaf, G.; Wolffenbuttel, R. F.; Correia, J. H. A Review of Visible-Range Fabry-Perot Microspectrometers in Silicon for the Industry. Opt. Laser Technol. 2012, 44 (7), 2312-2320.

(9) Dillon, P. L. P.; Brault, A. T.; Horak, J. R.; Garcia, E.; Martin, T. W.; Light, W. A. Fabrication and Performance of Color Filter Arrays for Solid- State Imagers. IEEE Trans. Electron Devices 1978, $25,97$.

(10) Chen, Q.; Hu, X.; Wen, L.; Yu, Y.; Cumming, D. R. S. Nanophotonic Image Sensors. Small 2016, 12 (36), 4922-4935.

(11) Macleod, H. Thin-Film Optical Filters, third ed.; Institute of Physics Publishing, 1986.

(12) Kristensen, A.; Yang, J. K. W.; Bozhevolnyi, S. I.; Link, S.; Nordlander, P.; Halas, N. J.; Mortensen, N. A. Plasmonic Colour Generation. Nat. Rev. Mater. 2017, 2 (1), 1-15.

(13) Shah, Y. D.; Grant, J.; Hao, D.; Kenney, M.; Pusino, V.; Cumming, D. R. S. Ultra-Narrow Line Width Polarization-Insensitive Filter Using a Symmetry-Breaking Selective Plasmonic Metasurface. ACS Photonics 2018, 5 (2), 663-669.

(14) Williams, C.; Rughoobur, G.; Flewitt, A. J.; Wilkinson, T. D. Nanostructured Plasmonic Metapixels. Sci. Rep. 2017, 7 (1), 7745.

(15) Kumar, K.; Duan, H.; Hegde, R. S.; Koh, S. C. W.; Wei, J. N.; Yang, J. K. W. Printing Colour at the Optical Diffraction Limit. Nat. Nanotechnol. 2012, 7 (9), 557-561.

(16) Burgos, S. P.; Yokogawa, S.; Atwater, H. A. Color Imaging via Nearest Neighbor Hole Coupling in Plasmonic Color Filters Integrated onto a Complementary Metal-Oxide Semiconductor Image Sensor. ACS Nano 2013, 7 (11), 10038-10047.

(17) Shaltout, A. M.; Kim, J.; Boltasseva, A.; Shalaev, V. M.; Kildishev, A. V. Ultrathin and Multicolour Optical Cavities with Embedded Metasurfaces. Nat. Commun. 2018, 9 (1), 1-7.

(18) Davis, M. S.; Zhu, W.; Xu, T.; Lee, J. K.; Lezec, H. J.; Agrawal, A. Aperiodic Nanoplasmonic Devices for Directional Colour Filtering and Sensing. Nat. Commun. 2017, 8 (1), DOI: 10.1038/s41467-01701268-y.

(19) Yokogawa, S.; Burgos, S. P.; Atwater, H. A. Plasmonic Color Filters for CMOS Image Sensor Applications. Nano Lett. 2012, 12 (8), 4349-4354. 
(20) Franklin, D.; Chen, Y.; Vazquez-Guardado, A.; Modak, S.; Boroumand, J.; Xu, D.; Wu, S. T.; Chanda, D. PolarizationIndependent Actively Tunable Colour Generation on Imprinted Plasmonic Surfaces. Nat. Commun. 2015, 6, 1-8.

(21) James, T. D.; Mulvaney, P.; Roberts, A. The Plasmonic Pixel:

Large Area, Wide Gamut Color Reproduction Using Aluminum Nanostructures. Nano Lett. 2016, 16 (6), 3817-3823.

(22) Jahani, S.; Jacob, Z. All-Dielectric Metamaterials. Nat. Nanotechnol. 2016, 11 (1), 23-36.

(23) Genevet, P.; Capasso, F.; Aieta, F.; Khorasaninejad, M.; Devlin, R. Recent Advances in Planar Optics: From Plasmonic to Dielectric Metasurfaces. Optica 2017, 4 (1), 139.

(24) Wang, P.; Menon, R. Ultra-High-Sensitivity Color Imaging via a Transparent Diffractive-Filter Array and Computational Optics. Optica 2015, 2 (11), 933.

(25) Kats, M. A.; Blanchard, R.; Genevet, P.; Capasso, F. Nanometre Optical Coatings Based on Strong Interference Effects in Highly Absorbing Media. Nat. Mater. 2013, 12 (1), 20-24.

(26) Li, Z.; Butun, S.; Aydin, K. Large-Area, Lithography-Free Super Absorbers and Color Filters at Visible Frequencies Using Ultrathin Metallic Films. ACS Photonics 2015, 2 (2), 183-188.

(27) Kajtár, G.; Kafesaki, M.; Economou, E. N.; Soukoulis, C. M. Theoretical Model of Homogeneous Metal-Insulator-Metal Perfect Multi-Band Absorbers for the Visible Spectrum. J. Phys. D: Appl. Phys. 2016, 49 (5), 55104.

(28) Diest, K.; Dionne, J.; Spain, M. Tunable Color Filters Based on Metal- Insulator- Metal Resonators. Nano Lett. 2009, 9 (7), 25792583.

(29) Williams, C.; Rughoobur, G.; Flewitt, A. J.; Wilkinson, T. D. Single-Step Fabrication of Thin-Film Linear Variable Bandpass Filters Based on Metal-Insulator-Metal Geometry. Appl. Opt. 2016, 55 (32), 9237.

(30) Grant, J.; Kenney, M.; Shah, Y. D.; Escorcia-Carranza, I.; Cumming, D. R. S. CMOS Compatible Metamaterial Absorbers for Hyperspectral Medium Wave Infrared Imaging and Sensing Applications. Opt. Express 2018, 26 (8), 10408.

(31) Frey, L.; Parrein, P.; Raby, J.; Pellé, C.; Hérault, D.; Marty, M.; Michailos, J. Color Filters Including Infrared Cut-off Integrated on CMOS Image Sensor. Opt. Express 2011, 19 (14), 13073.

(32) Yoon, Y.-T.; Lee, S.-S. Transmission Type Color Filter Incorporating a Silver Film Based Etalon. Opt. Express 2010, 18 (5), 5344.

(33) Noh, T. H.; Yoon, Y. T.; Lee, S. S.; Choi, D. Y.; Lim, S. C. Highly Angle-Tolerant Spectral Filter Based on an Etalon Resonator Incorporating a High Index Cavity. J. Opt. Soc. Korea 2012, 16 (3), 299-304.

(34) Frey, L.; Masarotto, L.; Melhaoui, L. E.; Verrun, S.; Minoret, S.; Rodriguez, G.; André, A.; Ritton, F.; Parrein, P. High-Performance Silver-Dielectric Interference Filters for RGBIR Imaging. Opt. Lett. 2018, 43 (6), 1355-1358.

(35) Yang, Z.; Chen, Y.; Zhou, Y.; Wang, Y.; Dai, P.; Zhu, X.; Duan, H. Microscopic Interference Full-Color Printing Using GrayscalePatterned Fabry-Perot Resonance Cavities. Adv. Opt. Mater. 2017, 5 (10), 1700029.

(36) Chen, Y.; Duan, X.; Matuschek, M.; Zhou, Y.; Neubrech, F.; Duan, H.; Liu, N. Dynamic Color Displays Using Stepwise Cavity Resonators. Nano Lett. 2017, 17 (9), 5555-5560.

(37) Wang, Y.; Zheng, M.; Ruan, Q.; Zhou, Y.; Chen, Y.; Dai, P.; Yang, Z.; Lin, Z.; Long, Y.; Li, Y.; et al. Stepwise-Nanocavity-Assisted Transmissive Color Filter Array Microprints. Research 2018, 2018, 110 .

(38) Lumerical Inc. www.Lumerical.Com/Tcad-Products/Fdtd/.

(39) Takigawa, T.; Kawabuchi, K.; Yoshimi, M.; Kato, Y. High Voltage Electron Beam Lithography. Microelectron. Eng. 1983, 1, 121142.

(40) Taguchi, H.; Enokido, M. Technology of Color Filter Materials for Image Sensor. Int. Image Sens. Work. 2011, 34-37. 